\title{
Nursing and Health Care
}

\section{The Epistemology of Swarming in Patient Care: An} Innovative Approach to Admitting Patients into Intensive Care Units

\section{Aretha D Miller ${ }^{1 *}$ and Cheryl Stephenson ${ }^{2}$}

\section{Affiliation}

${ }^{1}$ Nursing Department, Aspen University, USA

${ }^{2}$ Capella University, Greater Atlanta, USA

*Corresponding author: Aretha D Miller, Nursing Department, Aspen University, Denver, USA, E-mail: aretha.miller@aspen.edu

Citation: Miller DA and Stephenson C. The epistemology of swarming in patient care: an innovative approach to admitting patients into intensive care units (2021) Nursing and Health Care 5: 41-43.

Received: Dec 26, 2020

Accepted: Jan 21, 2021

Published: Jan 27, 2021

Copyright: ( 2021 Miller DA, et al. This is an open-access article distributed under the terms of the Creative Commons Attribution License, which permits unrestricted use, distribution, and reproduction in any medium, provided the original author and source are credited.

\begin{abstract}
Epistemology is the branch of philosophy that addresses cognitive sciences, the history of science and cultural studies. It investigates the origin, methods, nature and limits of knowledge and seeks to determine what we know and how we know it. It is also used to determine truth or falsehoods as we strive to acquire knowledge. This review examined the epistemology of Swarming in patient care, following the SQUIRE guidelines. No best practices exist on how to SWARM patients, yet this process is performed by frontline nurses daily. Four examples of Swarming were examined and a systematic approach on how to SWARM critically ill patients in the admission process into ICUs was recommended. Tasks needed once the patient arrives into the ICU suite are for the primary RN to receive bedside report from the healthcare team and $\log$ into the Electronic Health Record to start the admission note. A checklist was introduced to streamline tasks with the goal to reduce the number of chores to be performed by the primary RN, thus freeing up time for assessments of the critically ill patient. This work strives to improve efficiency in the workflow of the ICU admission process, by improving communication amongst the team via practice and a consistent approach to admitting critical patients.

Keywords: Swarming, Critical care, ICU, Admission.

Abbreviations: ICUs-Intensive Care Units, RCA-Root Cause Analysis, ED-Emergency Department, SCCM-Society for Critical Care Medicine, RNRegistered Nurse.
\end{abstract}

\section{Introduction}

Epistemology is the branch of philosophy that addresses cognitive sciences, the history of science and cultural studies. It investigates the origin, methods, nature, and limits of knowledge. It seeks to determine what we know, how we know it and is used to determine truth or falsehood as we seek to acquire knowledge of our world. Swarming in patient care made an initial impact in nursing when The University of Kentucky Healthcare in Lexington, UK created a standardized approach to investigate Root Cause Analysis (RCA) in 2009.

Since then, swarming in nursing is described in different ways. For instance, RCAs were referred to as Swarming. Another description of swarming was of a tool, which was designed to recognize inefficiencies in staff communication. Another example described Swarming as a swift evaluation of the patient by the healthcare team and the final description of SWARM was described as a type of gathering to discuss the events of a fall. Nevertheless, limited sources described events of swarming directly in nursing and the ones that did were difficult to access and not open source. To fill this gap, we presented a review of the various examples of swarming and recommended a novel approach of how swarming may be utilized in Intensive Care Units (ICUs) $[1,2]$.

Aim
We reviewed the research literature and examined the epistemology of swarming in patient care. The admission process of patients into ICUs can be busy to utterly chaotic at times. To the untrained eye, staff may be observed milling around in a small space trying to stabilize and provide care to patients. In order to provide some semblance of structure, to prevent duplication of tasks and to ensure required tasks for standard of care are completed, we recommended an innovative approach to assist the nursing staff when admitting critical patients into ICUs.

\section{Method}

Electronic databases CINAHL, MEDLINE, EMBASE, PsycINFO, ProQuest, PubMed and the Internet were searched using the terms "swarming" and "nursing". Although the recommendations are not a formal guideline, the AGREE checklist was used to develop this manuscript. No restrictions of published dates were placed. The target populations were patients and articles that discussed the use of swarming in patient care were selected. All articles that included swarming of insects were excluded. There were limited articles available on the use of swarming in patient care. Three articles were published by the Joint Commission, another by America's Essential Hospitals, and the next was as a letter to the editor of the Annals of

Citation: Miller DA and Stephenson C. The epistemology of swarming in patient care: an innovative approach to admitting patients into intensive care units (2021) Nursing and Health Care 5: 41-43. 
Emergency Nursing. A systematic review was conducted on these works and the examples outlined below.

\section{First Example of SWARM}

RCA is a universal approach used to investigate undesirable vulnerabilities in health care systems. From this research, RCAs were colloquially termed Swarming. The authors described five key steps to a SWARM. The process usually begins with an unwanted patient event; staff SWARMs to the site to determine cause or immediate corrective actions; an incident report is completed by the front-line worker; department heads review and evaluate the report as soon as possible; and then a meeting is set up for staff to determine what happened, why it happened and how to prevent the event from happening again see Figure 1. A meeting is then conducted in a neutral blame-free environment, introducing the process, the plan, purpose and the legal protections for staff who speak up. Everyone in the room is introduced, the facts of the incident are reviewed, discussion of the incident with any contributing factors and finally, proposed action plan, assignment of individuals to take ownership for additional investigations and implementation of a plan of action [2].

\section{Second Example of SWARM}

Williams et al., [3] used a quality improvement process to recognize, evaluate and encourage open dialogue with front-line workers to improve problem solving in a Pediatric ICU (PICU). The authors created a tool to empower caregivers to share information about problems or perceived problems and to quickly develop solutions in order to eliminate errors, process improvement and to develop a system of high reliability. The SWARM tool was designed to identify communication and inefficiencies in performance to develop an infrastructure of high reliability when managing problems. The approach to a problem specific SWARM begins by consulting upstream and downstream stakeholders in order to understand the scope of a problem. There is the use of an electronic tracking system to list the problems in categories, as well as possible solutions. Then, there is a process to disseminate learning opportunities to the involved caregivers on the specific units [3].

\section{Third Example of SWARM}

In a letter to the Editor of the Annals of Emergency Department (ED), Perniciaro and Liu described the redesign process of a Pediatric ED with upwards of 80,000 visits per year. The authors described Swarming as the instant evaluation of the patient together with the nurse, resident, and senior ED physician. Historically, in the ED a patient was initially assessed by the nurse, and then a medical assessment was performed by the physician.

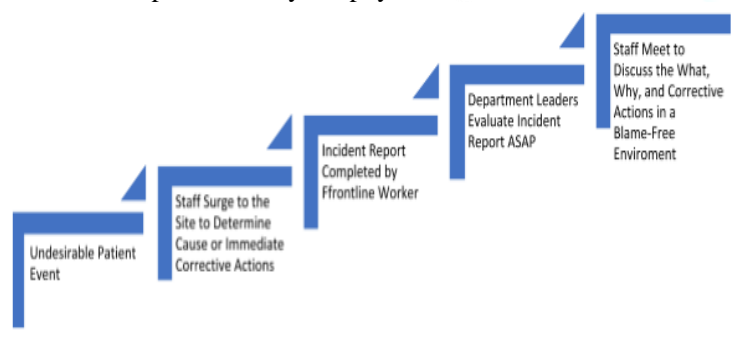

Figure 1: The Process of Swarming.

However, in their new model, patients were sorted quickly into tracks, placed in beds without the usual triage process, physicians were placed into zones, and patients were moved into treatments-in-progress and discharge areas. By eliminating the traditional serial workflow, efficiency was garnered via concurrent assessment of patients and education of residents. Using this process, this ED saw a drastic reduction in wait times and residents were able to model the senior medical staff. The authors asserted to have given birth to the concept of "swarming," and concluded that Swarming as a new process seem promising, but has several disadvantages including its efficacy and individual variability [4].

\section{Fourth Example of SWARM}

Motuel et al., [5] described a patient safety incident that occurred in a UK hospital. SWARM was described as a type of post incident huddle that was performed soon after the event in order to make improvements. SWARM was compared to RCAs as a superior tool because it stressed a blame-free culture with a focus on addressing the underlying issues. This example builds upon the standard approach and seminal work of $\mathrm{Li}$ et al, on how to investigate safety events that affect patients [2].

\section{Reasons for ICU Admissions}

The Society for Critical Care Medicine reported on the vital statistics of patients who end up in ICUs in the U.S. Nearly six million patients are admitted per year, needing invasive monitoring, airway support, and stabilization of life threatening conditions, management of illness or injuries, restoration of homeostasis, or care for the dying patient in a collaborative and interdisciplinary environment. About $20 \%$ of acute Care patients are admitted to ICUs, with approximately 58\% of ED patients resulting in ICU admissions. The most common ICU admissions are septicemia, percutaneous cardiovascular procedures, cerebral/myocardial infarction, and respiratory system diagnosis with up to $30 \%$ of patients requiring ventilatory support. The admission process of this patient population into ICUs can be busy to downright chaotic [6].

\section{Swarming in ICU Admissions}

Critically ill patients are often transported from EDs, Operating Rooms, other areas within a hospital system, or from Critical Care Transport. Generally, the receiving staff for a patient in the ICU include a Registered Nurse (RN), a respiratory therapist, and an Intensivist (physician who specializes in critical care medicine). In order to have the best outcomes, several actions are often performed simultaneously. During this period, an "all hands-on deck" approach is used where all available staff SWARM into the room to help. Nurses may be observed performing tasks like hooking the patient up to a cardiac monitor or trying to start peripheral intravenous lines.

There is usually no structure to these practices. Some would agree this is the culture of the ICU where nurses show up, lend a hand, and then return to their assigned patients. As the helpers are busy attempting to stabilize the patient, the primary RN should ensure the medications and equipment are verified for activation and correct settings. Some of the tasks needed once the patient arrives into the ICU suite are for the primary RN to receive a bedside report from the healthcare team, often using the framework called SBAR (Situation, Background, Assessment, Recommendation). Timely tasks include logging into the Electronic Health Record to start and or pend the admission note. Other simultaneous tasks are listed in the admission to ICU tasks checklist (Table 1) [7].

The assumption is the primary RN will be familiar with the healthcare team located in the ICU and feel comfortable with them to designate some of these tasks. Like processes used in the ED by Perniciaro and Liu to optimize clinical efficiencies, swarming could be used in ICUs to prevent duplication of tasks. We recommend the primary RN assign tasks to staff who show up to help. The checklist may be taped on the entrance door into the ICU and staff who complete a task may check the right-hand side of the checklist to express completion. Once the team returns to their designated areas, the primary RN will need to verify, but not have to spend a long time completing the tasks alone. This checklist may also be a good tool to communicate with oncoming staff at the change of shift. The checklist may be updated as needed by the nursing staff to fit their ICUs and to include tasks specific to their workflow [4]. 


\begin{tabular}{|c|c|}
\hline Tasks & $\begin{array}{c}\text { Check Once } \\
\text { Completed }\end{array}$ \\
\hline Summon the Intensivist to the room & \\
\hline Register new patient into the computer system & \\
\hline Register patient into the cardiac monitor & \\
\hline Transfer the patient onto the ICU bed & \\
\hline Apply telemetry monitoring equipment & \\
\hline $\begin{array}{c}\text { Full set of vital signs with temperature } \\
\text { Write temp on white board }\end{array}$ & \\
\hline $\begin{array}{c}\text { Double check drips with Medication Administration } \\
\text { Record } \quad \text { Untangle wires and add dates to tubing(s) }\end{array}$ & \\
\hline Chlorhexidine Gluconate (CHG) bath & \\
\hline $\begin{array}{c}\text { Remove old linen from underneath patient and } \\
\text { reposition patient }\end{array}$ & \\
\hline Weigh patient and write it on the white board & \\
\hline $\begin{array}{c}\text { Methicillin Resistant Staphylococcus Aureus swab, } \\
\text { print lab requisition, send to lab }\end{array}$ & \\
\hline $\begin{array}{c}\text { Hang Sequential Compression Device to the foot of } \\
\text { bed, apply compression sleeves to the patient's legs } \\
\text { and turn on machine }\end{array}$ & \\
\hline Update/Call family from Waiting Room \\
\hline Table 1: Admission to ICU Task Checklist.
\end{tabular}

Table 1: Admission to ICU Tasks Checklist.

\section{Limitations}

A standard definition of Swarming does not exist. Besides the benchmark study by $\mathrm{Li}$ et al., there is limited research on the use of Swarming in healthcare and no mention of its use in any ICUs. Swarming ICU patients would rely on the number of available staff to help to stabilize patients. When staff SWARM to a patient during the ICU admission process, the environment can seem chaotic, but quick assignment of roles before patient arrival or on arrival to the ICU may guide staff to be more efficient. The goal is to reduce the number of tasks for the primary $\mathrm{RN}$ to perform, freeing up time for continual assessment of the critically ill patient $[2,5]$.

\section{Conclusion}

The epistemology of Swarming in patient care revealed that it is a colloquial term created by a British hospital system in 2009 to describe RCAs. We described four examples of Swarming found in the nursing literature. Each example was unique and described measures to improve patient safety, and or ways to improve efficiencies in workflow through communication and performance. When the Swarming process was used in the ED, it helped to improve delays and prevented frustrations from patients answering repetitive questions. Similarly, Swarming a patient during the admission process in ICUs may improve communication amongst staff and help the primary RN to perform continual assessments in the stabilization of critical patients. No best practice exists on how to SWARM admitted patients into ICUs, but this process is performed by nurses daily and lacked a name until now. We believe the use of our checklist could enhance the ICU admission process and be used as a tool to streamline tasks, thus avoiding duplication and supporting the primary RN.

\section{References}

1. Bourgeois N. (2011). An epistemology of leadership perspective.

2. Li J, Boulanger B, Norton J, Yates A and Swartz. Swarming to improve patient care: A novel approach to root cause analysis (2015) Joint Commission J Qual Pati Saf 41: 494-501. https://doi.org/10.1016/S1553-7250(15)41065-7

3. Williams EA, Nikolai D, Ladwig L, Miller C and Fredeboelling, E. Development of swarm as a model for high reliability, rapid problem solving, and institutional learning. Joint Commission (2015) J Qual Pati Saf 41: 508-13.

https://doi.org/10.1016/j.evalprogplan.2008.08.009
4. Perniciaro J and Liu D. Swarming: A new model to optimize efficiency and education in an academic emergency department (2017) Annals Emerg Med 70: 435-436.

http://dx.doi.org/10.1016/j.annemergmed.2017.05.012

5. Motuel L, Dodds S, Jones S, Reid J and Dix A. Swarm: A quick and efficient response to patient safety incidents (2017) Nursing Times 113: 36-38.

6. Society of Critical Care Medicine (2019) Critical care statistics.

7. Liverpool Hospital (2017 February). ICU guideline: Clinical guidelines, receiving a patient into ICU. 